\title{
A Discrete Model for the Natural Frequencies and Mode Shapes of Constrained Vibrations of Beams with Various Boundary Conditions
}

\author{
A. RAHMOUNI ${ }^{1,2, a}, Z^{2}$ BEIDOURI ${ }^{1, b}$, and R. BENAMAR ${ }^{2, c}$ \\ 1 Laboratoire de Mécanique Productique et Génie Industriel (LMPGI) Université Hassan II Ain Chock, Ecole Supérieure \\ de Technologie, KM 7 Route El Jadida, B.P 8012 Oasis, Casablanca, Maroc ; \\ 2 Laboratoire des Etudes et Recherches en Simulation, Instrumentation et Mesures (LERSIM) Université Mohammed V - \\ Ecole Mohammadia des Ingénieurs, Avenue Ibn Sina, Agdal, Rabat, Maroc ;
}

\begin{abstract}
The purpose of the present paper is the development of a physically discrete model for free transverse constrained vibrations of beams. The discrete model consists on an $N$-degree of freedom system made of masses placed at the end of solid bars connected with spiral springs. The calculations made involve two tensors, namely the mass tensor $\left[m_{i j}\right]$ and the linear rigidity tensor $\left[k_{i j}\right]$. The results obtained by the physically discrete model show a good agreement and a quick convergence to the equivalent continuous beam for various end conditions for both the natural frequencies and the corresponding mode shapes. The model proposed in the present paper, which has been validated here using classical cases, may be easily applied to the flexural vibration of beams with various types of discontinuities, and to beams carrying concentrated masses.
\end{abstract}

\section{Introduction}

The purpose of the present paper is the development of a physically discrete model for the linear free transverse constrained vibrations of beams. The discrete model consists on an $\mathrm{N}$-degree of freedom system made of concentrated masses placed at the end of solid bars connected with spiral springs. Calculations are made using the mass tensor $\left[m_{i j}\right]$ and the linear rigidity tensor $\left[k_{i j}\right]$. The results obtained by the physically discrete model show a good agreement and a quick convergence to the equivalent continuous beam for various end conditions and for both the natural frequencies and the corresponding mode shapes. The model proposed in the present paper, which has been validated here using classical cases of beams with fixed ends, may be representative of various continuous beams with a localized nonlinearity, due for example, to a singularity in the mass or the elasticity distributions, and also to beams carrying concentrated masses.

\section{General theory}

\subsection{Presentation}

The $N$-dof system considered in the present work, made of $N$ masses $m_{1}, \ldots, m_{N}$ and $N+2$ spiral springs, is shown in Figure 1. $C_{r}$ being the linear rigidity of the spiral spring, for $r=1$ to $N+2$, the momentum $M_{r}$ in $C_{r}$ is given by: $M_{r}=-C_{r} \Delta \theta$. The spiral springs are connected by $N+1$ massless bars of length $l_{i}=l=\frac{L}{N+1}, i=1$ to $N+1$, where $\mathrm{L}$ is the total length of the bar $L=\sum_{i=1}^{N+1} l_{i}$.

\footnotetext{
a e-mail: abd.rahmouni@gmail.com

b e-mail: Zbeidouri@gmail.com

c e-mail: rbenamar@emi.ac.ma
}

Fig. 1. Multi-degree-of-freedom discrete system of masses with spiral springs

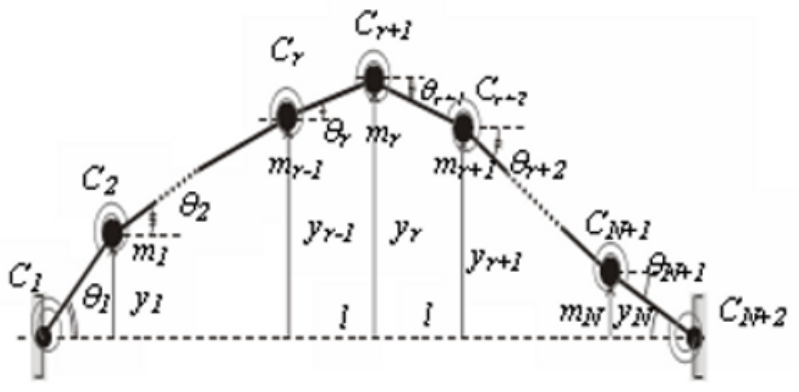

\subsection{Calculation of the coefficients $C_{i}$ in the discrete system representing the continuous beam}

The linear potential energy stored in the spiral spring $i$, subjected to the rotations shown in Figure 1, can be written as:

$$
V_{C_{i}}=\frac{1}{2} C_{i}\left(\theta_{i}-\theta_{i-1}\right)^{2} \quad \text { with } \quad \theta_{0}=\theta_{N+1}=0
$$

On the other hand, the elementary bending potential energy in a continuous beam is given by [1]:

$$
d V_{b}=\frac{1}{2} E I\left(\frac{d^{2} y}{d x^{2}}\right)^{2} d x
$$

In which $I$ is the quadratic moment relative to the neutral fibre of this section, $E\left(\mathrm{Nm}^{-2}\right)$ is the Young's modulus of the material. Using the finite difference technique, one can 
write:

$$
\frac{d^{2} y}{d x^{2}} / i=\frac{\frac{d y}{d x} / i-\frac{d y}{d x} / i-1}{l}=\frac{\theta_{i}-\theta_{i-1}}{l}
$$

By substituting equation (3) in equation (2), one obtains:

$$
d V_{b_{i}}=\frac{1}{2} \frac{E I}{l}\left(\theta_{i}-\theta_{i-1}\right)^{2}
$$

The identification between equations (1) and (4) leads to:

$$
C_{i}=\frac{E I}{l}=(N+1) \frac{E I}{L} \quad \forall i, 2 \leq i \leq N+1
$$

$C_{1}$ and $C_{N+2}$ are determined by the type of the constrained end conditions considered.

\subsection{Calculation of the stiffness matrix coefficients $k_{i j}$ of the discrete system}

In order to express the linear rigidity tensor general term $k_{i j}$ as a function of the beam characteristics, we recall the following expressions established in [2].

$$
\begin{gathered}
k_{r-2, r}=\frac{C_{r}}{l^{2}} \quad 3<r<N \\
k_{r-1, r}=-\frac{2}{l^{2}}\left(C_{r}+C_{r+1}\right) \quad r=2, \ldots, N \\
k_{r, r}=\frac{1}{l^{2}}\left(C_{r}+4 C_{r+1}+C_{r+2}\right) \quad r=1, \ldots, N
\end{gathered}
$$

Then, the coefficients of the stiffness matrix which are not dependent on the beam end conditions may be calculated as follows:

$$
\begin{array}{cc}
k_{r-2, r}=\frac{E I}{l^{3}} & 3<r<N \\
k_{r-1, r}=-4 \frac{E I}{l^{3}} & 2<r<N \\
k_{r, r}=6 \frac{E I}{l^{3}} & 2<r<N-1
\end{array}
$$

The other coefficients are obtained by symmetry or using the beam end conditions.

\subsection{General form of the stiffness matrix}

Factoring $(N+1)^{3} \frac{E I}{L^{3}}$ in the general term $k_{i j}$ of the stiffness matrix for a system with $N$ degrees of freedom leads to:

$$
\begin{aligned}
{\left[\mathbf{K}_{u v N}\right]=\frac{(N+1)^{3} E I}{L^{3}}\left(\begin{array}{cccccccccc}
Q_{1} & -4 & 0 & 0 & 0 & . & . & 0 & 0 & 0 \\
-4 & 6 & -4 & 1 & 0 & 0 & . & . & 0 & 0 \\
1 & -4 & 6 & -4 & 1 & 0 & 0 & . & . & . \\
0 & 1 & -4 & 6 & -4 & 1 & 0 & 0 & . & . \\
0 & 0 & . & . & . & . & . & . & . & . \\
. & . & . & . & . & . & . & . & . & . \\
. & . & . & . & . & . & . & . & . & . \\
. & . & . & . & 0 & 1 & -4 & 6 & . & . \\
. & . & . & . & . & 0 & 1 & -4 & 6 & -4 \\
0 & . & . & . & . & 0 & 0 & 1 & -4 & Q_{N}
\end{array}\right) } \\
=\frac{(N+1)^{3} E I}{L^{3}}\left[\mathbf{K}_{u v N}^{\prime}\right]
\end{aligned}
$$

The coefficients $Q_{1}$ and $Q_{N}$ depend on boundary conditions.

\subsection{Values of the stiffness matrix coefficients $Q_{1}$ and $Q_{N}$ for various end conditions}

Table 1 summarizes the values of the coefficients $C_{1}, C_{N+2}$, $Q_{1}$ and $Q_{N}$ that depend on the boundary conditions.

Table 1. : Coefficients of boundary conditions: S/S: Simply Supported beam; C/S: Clamped/Simply supported beam; C/C: Clamped-Clamped beam

\begin{tabular}{llll}
\hline & $\mathrm{S} / \mathrm{S}$ & $\mathrm{C} / \mathrm{S}$ & $\mathrm{C} / \mathrm{C}$ \\
\hline$C_{1}$ & 0 & $\infty$ & $\infty$ \\
$C_{N+2}$ & 0 & 0 & $\infty$ \\
$Q_{1}$ & 5 & $\infty$ & $\infty$ \\
$Q_{N}$ & 5 & 5 & $\infty$ \\
$u v$ & $\mathrm{ss}$ & $\mathrm{cs}$ & $\mathrm{cc}$ \\
\hline
\end{tabular}

\subsection{Calculation of the resonance frequency of the discrete system}

The eigenvalues of the non-dimensional rigidity matrix $\left[\mathbf{K}_{u v N}^{\prime}\right]$ will be computed numerically using Matlab's software to solve the eigenvalue problem :

$$
\left[\mathbf{K}_{u w N}^{\prime}\right]\{\mathbf{y}\}-\left(\omega_{\text {disc }}\right)^{2} \frac{\rho S L^{4}}{E I(N+1)^{4}}[\mathbf{I}]\{\mathbf{y}\}=\{\mathbf{0}\}
$$

where $\{\mathbf{y}\}$ is the displacement vector defined by: $\{\mathbf{y}\}^{T}=$ $\left\{y_{1} y_{2} \ldots y_{N}\right\}$, in which $y_{i}$ is the displacement of the $i^{\text {th }}$ mass and $[\mathbf{I}]$ is the identity matrix.

The resonance frequency corresponding to the $i^{\text {th }}$ mode of the discrete system is given by:

$$
\omega_{i}=\sqrt{\lambda_{i}}(N+1)^{2} \sqrt{\frac{E I}{\rho S L^{4}}}
$$

in which $\lambda_{i}$ is the $i^{t h}$ eigenvalue corresponding to the mass and rigidity matrices, and $\rho$ is the mass per unit volume of the bar in $\mathrm{kg} \mathrm{m} m^{-3}$. 


\section{Convergence in the case of a simply supported beam}

In the case of an $N$ dof system representing a simply supported beam, the rigidity matrix is given by:

$$
\begin{aligned}
& {\left[\mathbf{K}_{s s N}\right]=\frac{(N+1)^{3} E I}{L^{3}}\left(\begin{array}{cccccccccc}
5 & -4 & 1 & 0 & 0 & . & . & 0 & 0 & 0 \\
-4 & 6 & -4 & 1 & 0 & 0 & . & . & 0 & 0 \\
1 & -4 & 6 & -4 & 1 & 0 & 0 & . & . & . \\
0 & 1 & -4 & 6 & -4 & 1 & 0 & 0 & . & . \\
0 & 0 & . & . & . & . & . & . & . & . \\
. & . & . & . & . & . & . & . & . & . \\
. & . & . & . & . & . & . & . & . & . \\
. & . & . & . & 0 & 1 & -4 & 6 & -4 & 1 \\
. & . & . & . & . & 0 & 1 & -4 & 6 & -4 \\
0 & . & . & . & . & 0 & 0 & 1 & -4 & 5
\end{array}\right) } \\
&=\frac{(N+1)^{3} E I}{L^{3}}\left[\mathbf{K}_{s s N}^{\prime}\right]
\end{aligned}
$$

$\left[\mathbf{K}_{s s N}^{\prime}\right]$ is the non-dimensional linear rigidity matrix of a discrete system of $N$ masses and $N+1$ bars shown above. The eigenvalues of the matrix $\left[\mathbf{K}_{s s N}^{\prime}\right]$ have been computed numerically using Matlab's software. The values obtained have been then compared to the non-dimensional natural frequency $\omega_{c o n t}^{s s}$ of the corresponding continuous beam which are given by [1]:

$\omega_{\text {cont } 1}^{s s}=9.8696 ; \quad \omega_{\text {cont } 2}^{s s}=39.4784 ; \quad \omega_{\text {cont } 3}^{s s}=88.8264$

The results for $N=5,10,20,30$, and 100 are given in Table 2 and compared to the theoretical values of the SS continuous beam using the percentage error $\mathrm{p}(N)$ defined as:

$$
\mathrm{p}(N)=\frac{\omega_{\text {conti }}-\omega_{\text {disc }}(N)}{\omega_{\text {conti }}}
$$

Table 2. Comparison of the first, second and third linear frequencies of the $N$-dof system, for various values of $N$, with the the-

\begin{tabular}{|c|c|c|c|}
\hline$N$ and $\mathrm{p}(N)$ & $\begin{array}{l}\omega_{\text {disc } 1}^{s s} \\
1^{s t} \text { Mode }\end{array}$ & $\begin{array}{l}\omega_{\text {disc }}^{s s} \\
2^{\text {nd }} \text { Mode }\end{array}$ & $\begin{array}{l}\omega_{\text {disc } s}^{s s} \\
3^{\text {rd }} \text { Mode }\end{array}$ \\
\hline 5 & 9.6 & 36 & 72 \\
\hline $\mathrm{p}(5)$ & 0.03 & 0.09 & 0.2 \\
\hline 10 & 9.85 & 38.4 & 83.5 \\
\hline $\mathrm{p}(10)$ & 0.002 & 0.03 & 0.06 \\
\hline 20 & 9.87 & 39.18 & 87.4 \\
\hline $\mathrm{p}(20)$ & 0.0004 & 0.008 & 0.0016 \\
\hline 30 & 9.861 & 39.34 & 88.15 \\
\hline $\mathrm{p}(30)$ & 0.00009 & 0.004 & 0.008 \\
\hline 100 & 9.8688 & 39.466 & 88.76 \\
\hline $\mathrm{p}(100)$ & 0.00008 & 0.0003 & 0.0007 \\
\hline
\end{tabular}
oretical values of the frequencies of a SS continuous beam, and the percentage error $\mathrm{p}(N)$ obtained in each case.

It can be seen that there is a convergence of the linear frequency of the $N$-dof discrete system to that of a SS continuous beam when the number of masses $N$ increases. For example, a beam divided to 101 bars gives a relative error on the estimated frequency of $0.008 \%, 0.03 \%$ and $0.07 \%$ for the first, second and third mode respectively. Figures 2 ,
3 and 4 illustrate the convergence of the normalized first, second and third mode shape to that of the SS continuous beam [1].

Fig. 2. Convergence of the normalized first mode shape in the case of the simply/simply supported beam

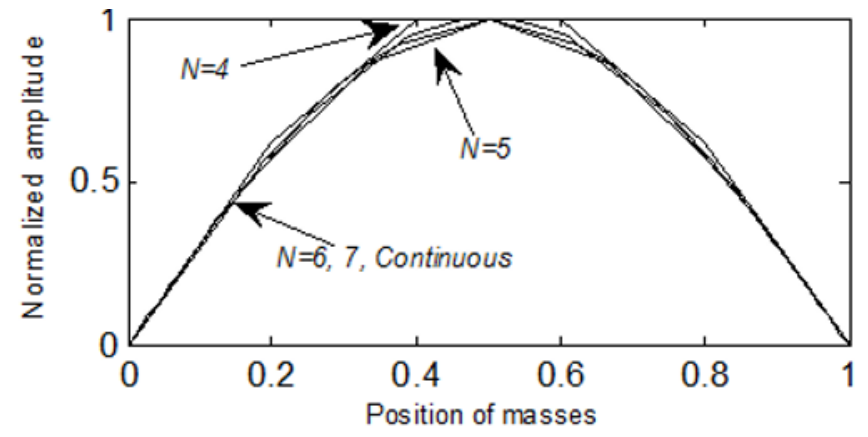

Fig. 3. Convergence of the normalized second mode shape in the case of the simply/simply supported beam

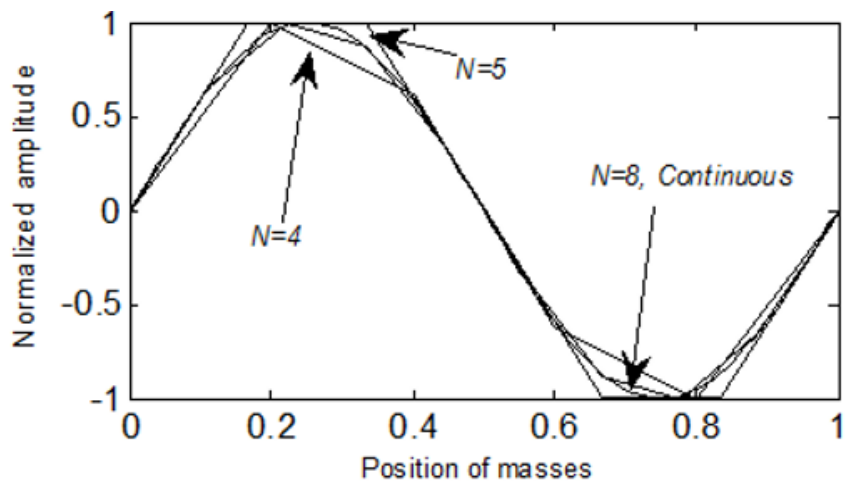

Fig. 4. Convergence of the normalized third mode shape in the case of the simply/simply supported beam

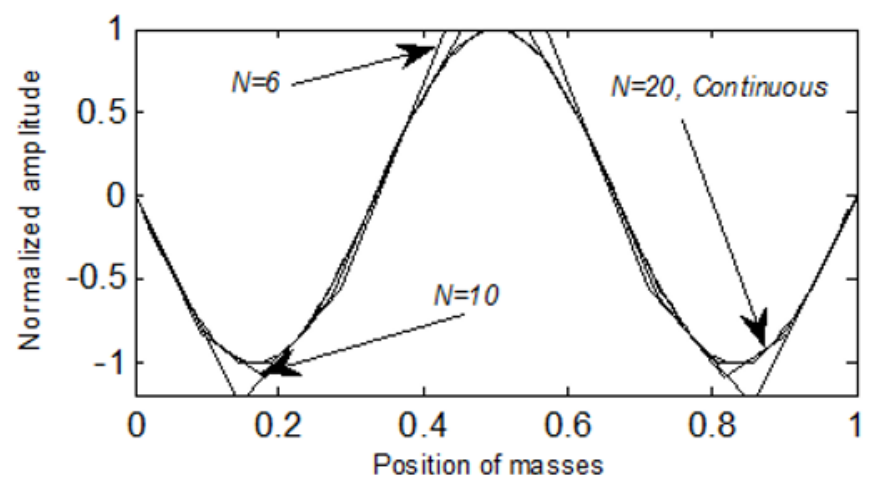

\section{Convergence in the case of a clamped/simply supported beam}

In the case of an $\mathrm{N}$-dof system representing a simply supported clamped beam, the rigidity matrix $\left[\mathbf{K}_{c s N}\right]$ is given 
by:

$$
\begin{aligned}
{\left[\mathbf{K}_{c s N}\right]=\frac{(N+1)^{3} E I}{L^{3}}\left(\begin{array}{cccccccccc}
\infty & -4 & 1 & 0 & 0 & . & . & 0 & 0 & 0 \\
-4 & 6 & -4 & 1 & 0 & 0 & . & . & 0 & 0 \\
1 & -4 & 6 & -4 & 1 & 0 & 0 & . & . & . \\
0 & 1 & -4 & 6 & -4 & 1 & 0 & 0 & . & . \\
0 & 0 & . & . & . & . & . & . & . & . \\
. & . & . & . & . & . & . & . & . & . \\
\cdot & . & . & . & . & . & . & . & . & . \\
. & . & . & . & 0 & 1 & -4 & 6 & -4 & 1 \\
. & . & . & . & . & 0 & 1 & -4 & 6 & -4 \\
0 & . & . & . & . & 0 & 0 & 1 & -4 & 5
\end{array}\right) } \\
=\frac{(N+1)^{3} E I}{L^{3}}\left[\mathbf{K}_{c s N}^{\prime}\right]
\end{aligned}
$$

$\left[\mathbf{K}_{c s N}\right]$ is the linear rigidity matrix of a discrete system of $N$ masses and $N+1$ bars representing a clamped simply supported beam of length $L=(N+1) l$. At the clamped end, the corresponding bar, which is bar $n^{\circ} 1$, cannot rotate, and consequently $C_{1}$ is infinite, which implies that $y_{1}=0$. This condition is taken into account numerically by suppressing the first line and first column of $\left[\mathbf{K}_{c s N}\right]$ and digitalizing the obtained reduced $(N-1)$ square matrix $\left[\mathbf{K}_{c s(N-1)}\right]$ defined by:

$$
\begin{gathered}
{\left[\mathbf{K}_{c s(N-1)}\right]=\frac{(N+1)^{3} E I}{L^{3}}\left(\begin{array}{cccccccccc}
6 & -4 & 1 & 0 & 0 & . & . & 0 & 0 & 0 \\
-4 & 6 & -4 & 1 & 0 & 0 & . & . & 0 & 0 \\
1 & -4 & 6 & -4 & 1 & 0 & 0 & . & . & . \\
0 & 1 & -4 & 6 & -4 & 1 & 0 & 0 & . & . \\
0 & 0 & . & . & . & . & . & . & . & . \\
. & . & . & . & . & . & . & . & . & . \\
. & . & . & . & . & . & . & . & . & . \\
. & . & . & . & 0 & 1 & -4 & 6 & -4 & 1 \\
. & . & . & . & . & 0 & 1 & -4 & 6 & -4 \\
0 & . & . & . & . & 0 & 0 & 1 & -4 & 5
\end{array}\right)} \\
=\frac{(N+1)^{3} E I}{L^{3}}\left[\mathbf{K}_{c s(N-1)}^{\prime}\right]
\end{gathered}
$$

The eigenvalues of the non-dimensional matrix $\left[\mathbf{K}_{c s(N-1)}^{\prime}\right]$ have been computed numerically using Matlab's software. The values obtained have been then compared to the nondimensional natural frequencies $\omega_{c o n t}^{c s}$ of the corresponding continuous clamped simply supported beam which are given by [1]:

$\omega_{\text {cont } 1}^{c s}=15.4181 ; \quad \omega_{\text {cont } 2}^{c s}=49.9651 ; \quad \omega_{\text {cont } 3}^{c s}=104.25$

The results for $N=5,10,20,30,100$ and 1000 are given in Table 3 and compared to the theoretical values of the CS continuous beam showing a good convergence.

Figures 5, 6 and 7 illustrate the convergence of respectively the normalized first, second and third mode shape to that of the continuous beam.
Table 3. Comparison of the first, second and third linear frequen-

\begin{tabular}{|c|c|c|c|}
\hline$N$ and $\mathrm{p}(N)$ & $\begin{array}{l}\omega_{\text {disc } 1}^{c s} \\
1^{s t} \text { Mode }\end{array}$ & $\begin{array}{l}\omega_{\text {disc }}^{c s} \\
2^{\text {nd }} \text { Mode }\end{array}$ & $\begin{array}{l}\omega_{\text {disc }}^{c s} \\
3^{\text {rd }} \text { Mode }\end{array}$ \\
\hline 5 & 18.1 & 54.2 & 96.9 \\
\hline $\mathrm{p}(5)$ & -0.174 & -0.085 & 0.071 \\
\hline 10 & 16.9 & 53.6 & 107.8 \\
\hline $\mathrm{p}(10)$ & -0.096 & -0.073 & -0.034 \\
\hline 20 & 16.2 & 52.1 & 107.8 \\
\hline $\mathrm{p}(20)$ & -0.051 & -0.043 & -0.034 \\
\hline 30 & 15.9 & 51.5 & 107 \\
\hline $\mathrm{p}(30)$ & -0.031 & -0.031 & -0.026 \\
\hline 100 & 15.6 & 50.5 & 105.2 \\
\hline $\mathrm{p}(100)$ & -0.012 & -0.011 & -0.009 \\
\hline 1000 & 14.434 & 50.015 & 104.35 \\
\hline $\mathrm{p}(1000)$ & -0.001 & -0.001 & -0.001 \\
\hline
\end{tabular}
cies of the $N$ dof system, for various values of $N$, with the theoretical values of the frequencies of a CS continuous beam, and the percentage error $\mathrm{p}(N)$ obtained in each case.

Fig. 5. Convergence of the normalized first mode shape in the case of the clamped/simply supported beam

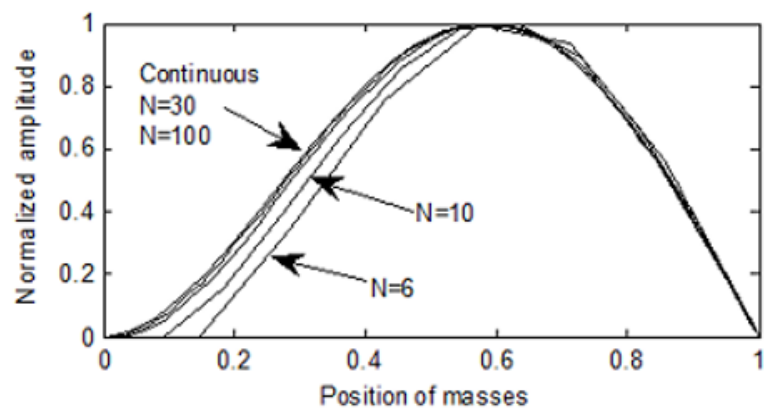

Fig. 6. Convergence of the normalized second mode shape in the case of the clamped/simply supported beam

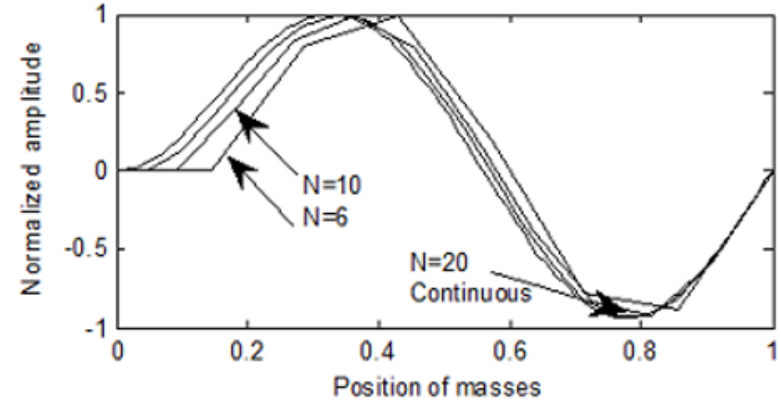

Fig. 7. Convergence of the normalized third mode shape in the case of the clamped/simply supported beam

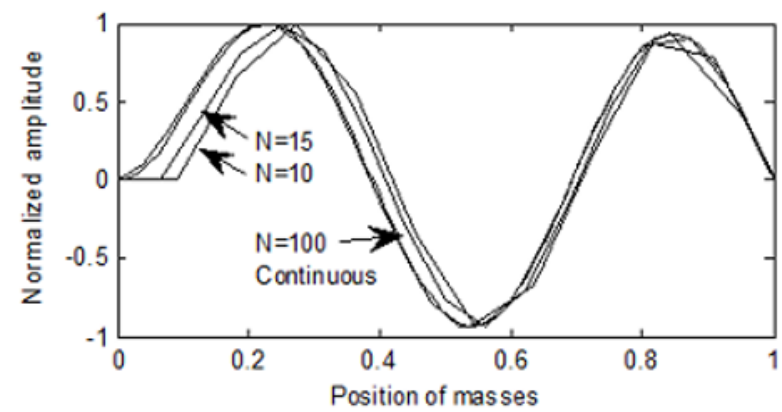




\section{Convergence in the case of a beam clamped at both sides}

In the case of an $N$-dof system representing a clamped clamped beam, the rigidity matrix $\left[\mathbf{K}_{c c N}\right]$ is given by:

$$
\begin{aligned}
& {\left[\mathbf{K}_{c c N}\right]=\frac{(N+1)^{3} E I}{L^{3}}\left(\begin{array}{cccccccccc}
\infty & -4 & 1 & 0 & 0 & . & . & 0 & 0 & 0 \\
-4 & 6 & -4 & 1 & 0 & 0 & . & . & 0 & 0 \\
1 & -4 & 6 & -4 & 1 & 0 & 0 & . & . & . \\
0 & 1 & -4 & 6 & -4 & 1 & 0 & 0 & . & . \\
0 & 0 & . & . & . & . & . & . & . & . \\
. & . & . & . & . & . & . & . & . & . \\
. & . & . & . & . & . & . & . & . & . \\
. & . & . & . & 0 & 1 & -4 & 6 & -4 & 1 \\
. & . & . & . & . & 0 & 1 & -4 & 6 & -4 \\
0 & . & . & . & . & 0 & 0 & 1 & -4 & \infty
\end{array}\right) } \\
&=\frac{(N+1)^{3} E I}{L^{3}}\left[\mathbf{K}_{c c N}^{\prime}\right]
\end{aligned}
$$

$\left[\mathbf{K}_{c c N}\right]$ is the linear rigidity matrix of a discrete system of $N$ masses and $N+1$ bars representing a clamped clamped beam of length $L=(N+1) l$. At the clamped ends, the corresponding bars, which are bars $\mathrm{n}^{\circ} 1$ and $\mathrm{n}^{\circ}(N+1)$, can not rotate, and consequently $C_{1}$ and $C_{N+2}$ are infinite, which implies that $y_{1}=0$ and $y_{N}=0$. These two conditions are taken into account numerically by suppressing the first and the last lines and the first and the last columns of [ $\left.\mathbf{K}_{c c N}\right]$ and diagonalizing the obtained reduced $(N-2)$ square matrix $\left[\mathbf{K}_{c c(N-2)}\right]$ defined by:

$$
\begin{aligned}
{\left[\mathbf{K}_{c c(N-2)}\right]=\frac{(N+1)^{3} E I}{L^{3}}\left(\begin{array}{cccccccccc}
6 & -4 & 1 & 0 & 0 & . & . & 0 & 0 & 0 \\
-4 & 6 & -4 & 1 & 0 & 0 & . & . & 0 & 0 \\
1 & -4 & 6 & -4 & 1 & 0 & 0 & . & . & . \\
0 & 1 & -4 & 6 & -4 & 1 & 0 & 0 & . & . \\
0 & 0 & . & . & . & . & . & . & . & . \\
. & . & . & . & . & . & . & . & . & . \\
. & . & . & . & . & . & . & . & . & . \\
. & . & . & . & 0 & 1 & -4 & 6 & -4 & 1 \\
. & . & . & . & . & 0 & 1 & -4 & 6 & -4 \\
0 & . & . & . & . & 0 & 0 & 1 & -4 & 6
\end{array}\right) } \\
= \\
=\frac{(N+1)^{3} E I}{L^{3}}\left[\mathbf{K}_{c c(N-2)}^{\prime}\right]
\end{aligned}
$$

The eigenvalues of the matrix $\left[\mathbf{K}_{c c(N-2)}^{\prime}\right]$ have been computed numerically using Matlab's software. The values obtained have been then compared to the non-dimensional natural frequencies $\omega_{c o n t}^{c c}$ of the corresponding continuous clamped clamped beam which are given by [1]:

$\omega_{\text {cont } 1}^{c c}=22.373 ; \quad \omega_{\text {cont } 2}^{c c}=61.673 ; \quad \omega_{\text {cont } 3}^{c c}=120.903$

The results obtained for $N=5,10,20,30$ and 100 are given in table 4 and compared to the theoretical values of the CC continuous beam showing a good convergence.

It can be seen that there is a convergence of the linear frequency to that of a continuous beam when the number of masses $N$ increases. For example, a beam divided to 101 bars gives a relative error of $2 \%$. Figures 8,9 and 10 illustrate the convergence of the normalized first, second and third mode shape of the discrete system to that of the continuous beam.
Table 4. Comparison of the first, second and third linear frequen-

\begin{tabular}{|c|c|c|c|}
\hline$N$ and $\mathrm{p}(N)$ & $\begin{array}{l}\omega_{\text {discl }}^{c c} \\
1^{s t} \text { Mode }\end{array}$ & $\begin{array}{l}\omega_{\text {disc2 }}^{c c} \\
2^{\text {nd }} \text { Mode }\end{array}$ & $\begin{array}{l}\omega_{\text {disc3 }}^{c c} \\
3^{r d} \text { Mode }\end{array}$ \\
\hline 5 & 32.6 & 80.5 & 125.6 \\
\hline $\mathrm{p}(5)$ & -0.457 & -0.305 & -0.039 \\
\hline 10 & 27.2 & 73.5 & 138.4 \\
\hline $\mathrm{p}(10)$ & -0.216 & -0.192 & -0.145 \\
\hline 20 & 24.7 & 67.8 & 131.7 \\
\hline $\mathrm{p}(20)$ & -0.104 & -0.099 & -0.089 \\
\hline 30 & 23.9 & 65.8 & 128.4 \\
\hline $\mathrm{p}(30)$ & -0.068 & -0.067 & -0.062 \\
\hline 100 & 22.82 & 62.9 & 123.3 \\
\hline $\mathrm{p}(100)$ & -0.020 & -0.020 & -0.020 \\
\hline
\end{tabular}
cies of the $N$-dof system, for various values of $N$, with the theoretical values of the frequencies of a CC continuous beam, and the percentage error $\mathrm{p}(N)$ obtained in each case.

Fig. 8. Convergence of the normalized first mode shape in the case of the clamped/clamped beam

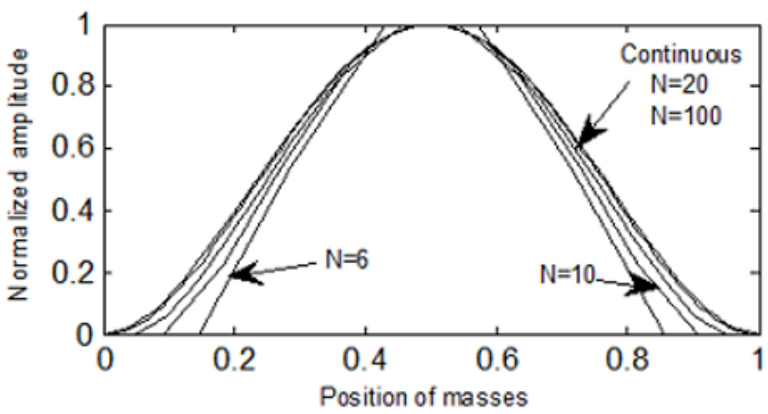

Fig. 9. Convergence of the normalized second mode shape in the case of the clamped/clamped beam

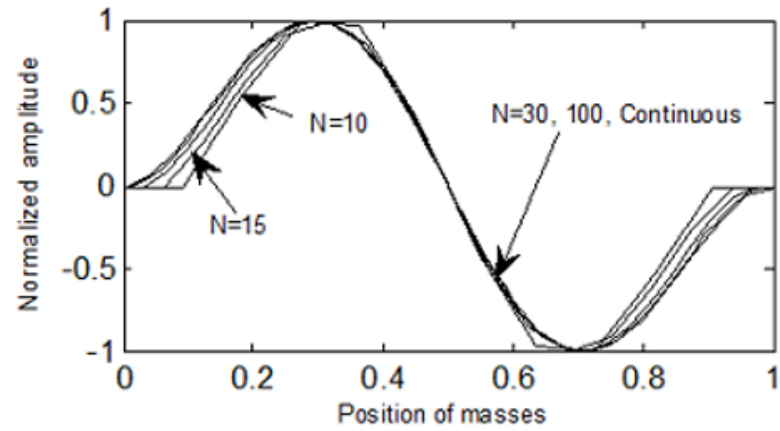

Fig. 10. Convergence of the normalized third mode shape in the case of the clamped/clamped beam

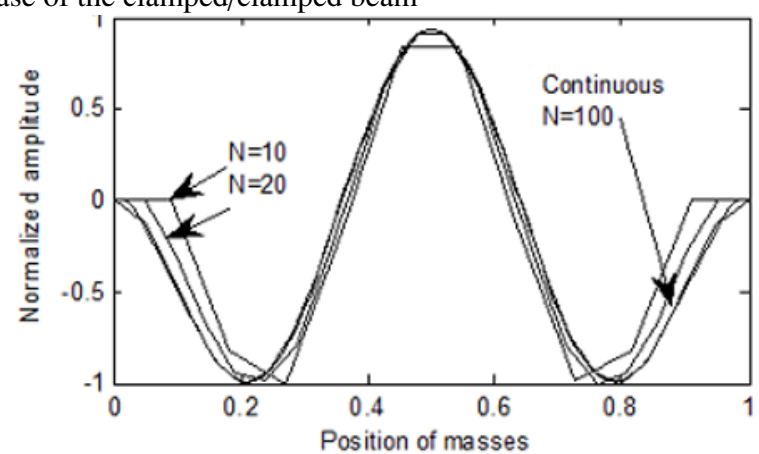

\section{Conclusion}

A physical discrete model for linear transverse vibrations of beams has been developed with the objective of pre- 
senting the continuous beam by a series of concentrated masses, connected by bars. In the nodes, torsional springs have been placed to present the flexural rigidity of the beam. The results obtained for the linear frequencies of the discrete system compare well with the analogous continuous beam. The model proposed in reference [1] has been completed by developing the discrete continuous analogy. On the other hand, the model proposed in the present paper, shown in Figure 1, may be representative of the flexural vibration of a beam with various types of discontinuities. The values of the torsional stiffness $C_{1}, C_{2}, \ldots, C_{N+1}$ of springs $2,3 \ldots, N+1$ and the masses $m_{1}, m_{2}, \ldots, m_{N}$ may be adapted, depending on the type of singularities considered, such as concentrated masses or various types of junctions along the beam. Concerning the values of the stiffness $C_{1}$ and $C_{N+2}$ of the torsional springs 1 and $N+2$, located at the two beam ends, they represent the nature of the end conditions, i.e. clamped or simply supported. It is also possible to use the present model for presenting beams with flexural constraints at the end. On the other hand, identification procedures may be easily implemented using the present model in order to estimate the stiffness of the real boundaries of a tested beam using experimental data. However, one must be cautious with respect to the limits of validity of the model that may not be appropriate to characterize junctions with a structural complexity. Previous studies have shown in such cases many difficulties in comparing theoretical models with experimental results $[3,4]$.

\section{References}

1. Thomson W.T. Dahleh M.D, Theory of vibrations with applications (Prentice-Hall, Upper Slide River, 1998), 438-448

2. A.Eddanguir, Geometrically Non-linear Transverse Vibrations of Discrete Multi-Degrees of Freedom Systems with a Localised Non-Linearity, International Journal of Mathematics and Statistics, 4, (2009) Number S09

3. E Savin, Mid-frequency vibrations of a complex structure: Experiments and comparison with numerical simulations. AIAA Journal, 40, $\mathrm{N}^{\circ}$ 9, September (2002), 1876-1884.

4. Soize, Reduced models for structures in the mediumfrequency range coupled with internal acoustic cavities. Journal of the Acoustical Society of America, 106, $n^{\circ}$ 6, December (1999), 3362-3374. 